\title{
Copy number variations' effect on drug response still overlooked
}

People vary widely in how they respond to medications, in part because of differences in their genetic makeup. Decades of research have helped untangle how slight variations in our DNA have this effect, fostering the advent of 'pharmacogenetics.' But scientists have largely overlooked the influence of gene duplications or deletions-called copy number variations-on drug response.

"The highest-level science has not yet been applied to copy number in the context of drugs," says Howard McLeod, medical director of the DeBartolo Family Personalized Medicine Institute at the University of South Florida Moffitt Cancer Center in Tampa. "We really haven't tried hard enough."

New insights could draw more attention to copy number variations, also known as CNVs. A study published in January reports that CNVs occur not only in drug metabolism genes, but also in genes that regulate the uptake of drugs into cells. A team of Korean researchers examined 152 genes in tissue and blood samples from people diagnosed with cancer affecting the liver, lungs, blood or lymph nodes. They found CNVs in more than ten genes in normal blood and tissue, including an extra copy of a key transporter for some antiviral and anticancer drugs (Kim, I.W. et al., Pharmacogenet. Genomics 25, 1-7, 2015). The team also reported a greater frequency of CNVs in certain cancer tissues compared with normal samples, including variations in a few genes that affect how cancer drugs are trafficked in and out of cells (although the paper did not examine the relationship between CNVs and drug response). Other groups have identified CNVs in a handful of other genes, but there may be many more that haven't been discovered.

People typically carry two copies of each gene (one from each parent), but copies can be gained or lost. These variations can have a profound-and sometimes deadly-impact. In 2005, a woman who had recently given birth began taking codeine for postpartum pain. Codeine works because an enzyme called CYP2D6 converts the medication to morphine, a painkiller. Because the woman metabolized the drug too quickly, morphine levels in her body-and her breast milkspiked. She survived the episode, but her infant did not. Only after her two-week-old son died did researchers discover that she carried three copies of the CYP2D6 gene, making her an 'ultra-rapid metabolizer.'

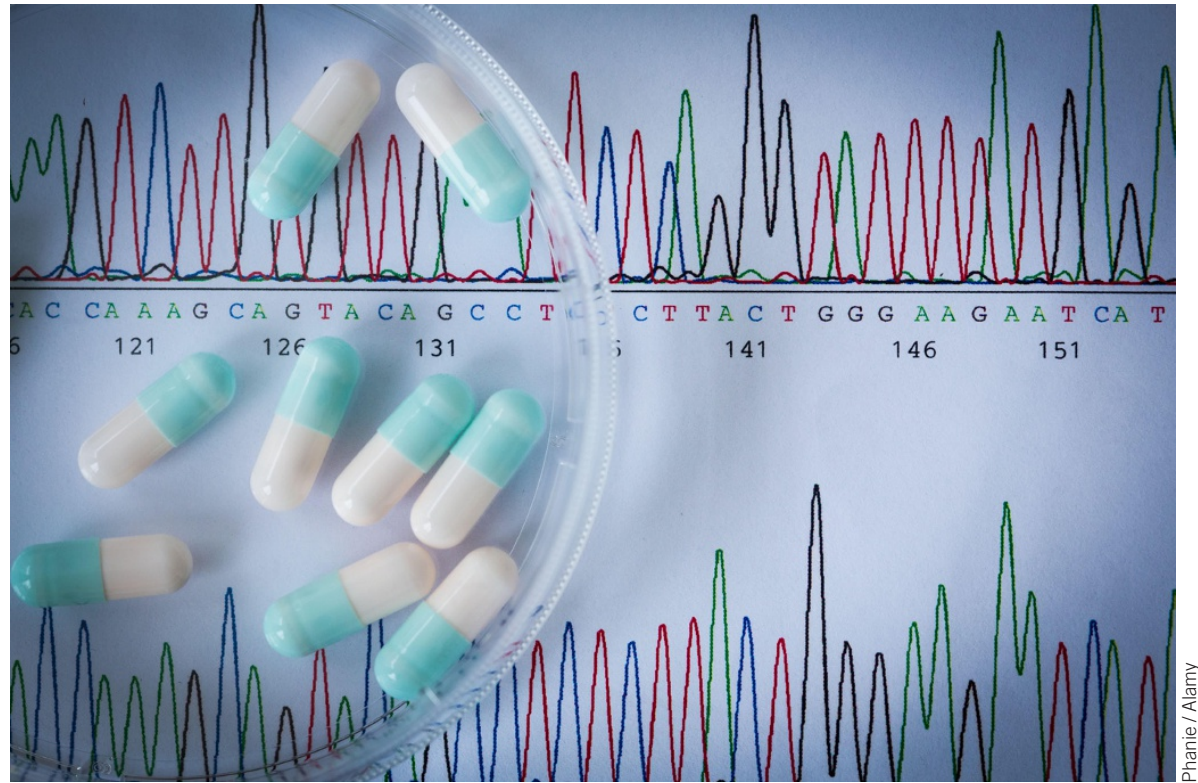

Seeing double: Having multiple copies of a gene can influence results.

CYP2D6 copy number variations can also affect how people respond to about 30 other drugs, including other opioids, tamoxifen and antipsychotics.

\section{Counting copies}

The consequences of CNVs in other genes is less clear, in part because pharmacogenomic researchers haven't devoted as much effort to studying these structural variations as they have to assessing the impact of base changes known as single-nucleotide polymorphisms (SNPs). “They're a lot more complex and harder to genotype than SNPs are," says Charles Lee, scientific director of the Jackson Laboratory for Genomic Medicine in Farmington, Connecticut. And the chip technology researchers use to find SNPs isn't always well suited to pinpointing copy number variants. Even whole-genome sequencing techniques can't provide accurate copy number data-at least not yet. "Sequencing technology has really blossomed in the last few years," says Stuart Scott, who studies pharmacogenomics at the Icahn School of Medicine at Mount Sinai in New York. "The ability to infer copy number from next-generation sequencing is going to completely change."

In the clinic, testing for CNVs is rare. The US Food and Drug Administration (FDA) requires doctors to order genotyping of CYP2D6 in two instances: when they prescribe eliglustat, a new pill for one form of Gaucher disease, or thioridazine, an antipsychotic available for compassionate use. The agency also recommends CYP2D6 genotyping for high daily doses of tetrabenazine, a drug used to treat a movement disorder caused by Huntington disease, as well as for high doses of the antipsychotic medication pimozide, which is used to treat symptoms of Tourette syndrome.

The two FDA-approved tests for CYP2D6 genotyping-Roche's AmpliChip CYP450 test and Luminex's xTAG CYP2D6 kit-do look for CNVs, but some other laboratorydeveloped tests, which are not yet regulated by the FDA, do not. And even tests that do examine copy number may not drill down further to assess whether extra gene copies are functional. "A lot of these commercial platforms do not discriminate," says Andrea Gaedigk, director of the Pharmacogenetics Laboratory at Children's Mercy Hospital in Kansas City, Missouri. "There are so many laboratories out there right now who perform testing, and there are no standards of how many variants should even be tested."

Minoli Perera, a pharmacogenomics expert at the University of Chicago, says that interest in $\mathrm{CNV}$ and drug response is growing. And Perera thinks this kind of variation is likely to be important. With respect to drug response, "we're certainly not capturing all of the variability that should be attributed to genetics," she says. "There have to be other things that we're overlooking, and copy number may be one of those."

Cassandra Willyard 